\title{
Chroniques génomiques
}

\section{Génotypage tous azimuts}

\section{Bertrand Jordan}

Après quelques années de relative stabilité, les techniques d'analyse du génome ont fait un nouveau bond en avant. Le séquençage s'affranchit enfin de la bonne vieille méthode de Sanger (1977...) et les nouvelles machines «massivement parallèles» lisent plusieurs mégabases par jour. Les microarrays débordent de leur cadre initial, l'étude de l'expression, et permettent aujourd'hui (entre autres) le génotypage de milliers d'individus pour des centaines de milliers de Snips (single nucleotide polymorphism). Du coup, les études d'association à grande échelle pour tenter d'identifier des gènes impliqués dans des maladies multigéniques se multiplient, et la perspective d'une connaissance détaillée du génome de chacun d'entre nous se rapproche, pour le meilleur et peut-être pour le pire.

Évidemment l'industrie n'est pas en reste, comme en témoigne la création d'une société curieusement appelée $23 a n d M e^{1}$ dont le slogan est «La génétique va devenir personnelle » et qui se propose de vous offrir «un accès détaillé, sécurisé et privé à votre information génétique $»^{2}$. Fondée l'an dernier, l'entreprise a réussi son premier «tour de table » en mai 2007, avec des investisseurs comme Genentech et Google. La part de Google, qui est investisseur minoritaire, s'élève à près de quatre millions de dollars: le total (non communiqué) doit se situer entre dix et vingt millions, un

${ }^{1}$ Le « 23 » se réfère en fait à nos vingt-trois chromosomes, et le nom de l'entreprise signifie donc à peu près « Mon génome et moi »...

${ }^{2}$ http://www.23andme.com/

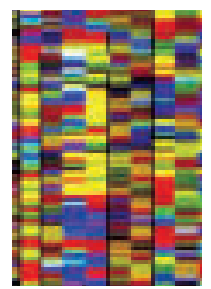

Marseille-Nice Génopole, case 901 ,

Parc Scientifique de Luminy, 13288 Marseille Cedex 9, France. brjordan@club-internet.fr

beau chiffre pour une start-up ne disposant d'aucune technologie en propre... L'aventure semble donc être prise au sérieux par le monde de la biotech américaine. D'ailleurs, 23 andMe a récemment signé un partenariat avec Illumina, l'entreprise qui concurrence sérieusement Affymetrix sur le marché de la mesure d'expression et du génotypage ${ }^{3}$, afin de développer le marché du consumer genotyping. L'objectif est de «permettre aux individus d'obtenir des informations détaillées sur leur ascendance, leur généalogie et leurs caractéristiques héréditaires ». Visiblement, les experts pensent qu'il existe une demande pour ce type d'information, et le PDG d'Illumina déclarait récemment que «cela va être un marché très important, et cela pourrait arriver très vite $»^{4}$.

Notre génome devient donc personnel, comme le dit $23 a n d M e$, il devient surtout commercial. Les experts en bio-éthique dont l'entreprise affirme s'entourer vont avoir fort à faire s'ils veulent vraiment éviter les dérives et faire en sorte que cette information génétique soit employée pour aider les individus et non au bénéfice des employeurs ou des compagnies d'assurance... $\diamond$ Genotyping everywhere

\footnotetext{
${ }^{3}$ Chiffre d'affaires 2007 d'lllumina : plus de trois cent millions de dollars.

${ }^{4}$ «Our anticipation is that it is going to be a very large market, and that it could be a large market very quickly».
}

\section{TIRÉS À PART}

B. Jordan 